\title{
THE CONCEPT OF LIDAR DATA QUALITY ASSESSMENT IN THE CONTEXT OF BIM MODELING
}

\author{
A. Warcho1 $1^{1,2}$ \\ ${ }^{1}$ State Higher School of Technology and Economics in Jarosław, Poland \\ ${ }^{2}$ ProGea 4D sp. z o.o. Krakow, Poland \\ artur_warchol@o2.pl
}

Commission I, WG I/10

KEY WORDS: laser scanning, LiDAR, BIM, data quality, point clouds

\begin{abstract}
:
LiDAR technology has revolutionized the area of 3D data acquisition. It is possible to obtain in a very fast and accurate way geometric and other information for a large area. Along with the development of design technology, LiDAR point clouds are often used to create inventory models of building objects and installations. This paper presents the possibilities of assessing LiDAR data for BIM modeling. The areas in which the assessment and description of obtained TLS data is important are presented. In addition to the attributes for assessing the quality of spatial data contained in the ISO 19157 standard, a density parameter was proposed. Examples of point clouds with different density for the architectural detail are presented in the final part of the work. For the attributes describing LiDAR data sets the levels of importance have been proposed for.
\end{abstract}

\section{INTRODUCTION}

The development of measurement technologies allows to register the space around us more rapidly and accurately. One of the most modern technologies is LiDAR (Light Detection and Ranging), i.e. measurement with a laser beam. Regardless of the platform on which the scanner is located, the result of the measurement is the point cloud, that is the set of coordinates (X, Y, Z at the beginning and $\mathrm{X}, \mathrm{Y}, \mathrm{H}$ after georeferencing) along with additional information - reflection intensity, RGB values, echo number, etc. Depending on the placement of the measuring instrument, we can distinguish the following methods of scanning:

- SLS (Satellite Laser Scanning)

- ALS (Airborne Laser Scanning) - with a plane, helicopter and UAV (Unmanned Aerial Vehicle),

- MLS (Mobile Laser Scanning) - with a car, trolley, backpack or a device carried in the hands of the operator (ZEB - Horizon)

- TLS (Terrestrial Laser Scanning) - on a tripod.

The accuracy of the acquired data and the density per unit area increases with the decreasing height of acquisition. Unfortunately, at the same time the rate of data acquisition for the same unit of area decreases as well.

In most cases, TLS data rather than MLS or ALS-UAV data are used for the registration of the surrounding space in LiDAR technology in the context of modeling BIM (Building Information Modeling) objects. Due to the characteristics of LiDAR technology, it is usually used in the context of BIM for obtaining information about the geometry of objects. Inventories of objects prepared on the basis of LiDAR point clouds allow to create 2D-CAD documentation, 3D models or 3D-BIM models.

The quality of LiDAR data is extremely important when point clouds are used for inventory. It has a direct impact on the quality of the final documentation, and in extreme cases, it is even impossible to make documentation based on poor LiDAR data. Therefore, it is essential that the acquired point clouds meet the quality parameters allowing its use for the planned aims.

The purpose of acquiring data and planned LoD (Level of Detail) of the BIM model being developed determines the quality parameters of point clouds.

Due to the lack of guidance in the field of LiDAR TLS data control, it is recommended to apply some of the guidelines for INIK (Inspector of Supervision and Control) in the ISOK project, i.e. "IT System for Protecting the Country against Extraordinary Dangers" (GUGiK, 2011, GUGiK, 2013).

\section{THE ISOK PROJECT}

The ISOK project was implemented in the years $2010-2015$ by a consortium: The Institute of Meteorology and Water Management - National Research Institute (IMGW-PIB) (leader), National Water Management Board (KZGW), Head Office of Geodesy and Cartography (GUGiK), The National Institute of Telecommunications - the State Research Institute (It-PIB) and the Government Centre for Security (RCB) as a supporting body. The main goal of the project was to create a new element significantly improving the protection of society, economy and the environment against the effects of floods and other extraordinary threats. The surveying part of the project was managed by GUGiK, and it consisted of 4 detailed tasks.

Task 1 - obtaining altitude data in the technology of Airborne Laser Scanning (ALS) for river basins with a total target area of about $289000 \mathrm{~km}^{2}$. Products delivered to the GUGiK were:

- Product 1 - laser point clouds in Standard I:

- for an area of $267403 \mathrm{~km}^{2}$ with a density of 4 points $/ \mathrm{m}^{2}$.

- for an area of $8148 \mathrm{~km}^{2}$ with a density of 6 points $/ \mathrm{m}^{2}$ (priority areas)

- Product 2 - Standard II laser point cloud for $13769 \mathrm{~km}^{2}$ of urban areas with a density of 12 points $/ \mathrm{m}^{2}$. 
In addition, Products 1 and 2 will have RGB attributes and intensity.

- Product 3 - Digital Terrain Model in a uniform standard for the entire study $289000 \mathrm{~km}^{2}$ - with 1m GRID.

- Product 4 - Digital Surface Model for the area of $275551 \mathrm{~km}^{2}$ - with $1 \mathrm{~m}$ GRID (corresponds to the coverage of Product 1)

- Product 5 - Digital Surface Model for the area of $13769 \mathrm{~km}^{2}$ - with $0.5 \mathrm{~m}$ GRID (corresponds to the coverage of Product 2)

- Product 6 - aerial imaging as an auxiliary interpretative material and source of information about RGB attributes for the LIDAR point cloud, GSD $0.5 \mathrm{~m}$.

Task 2 - developing the concept and implementing the Digital Terrain Model Management System.

Task 3 - developing a digital orthophotomap covering an area of 203 cities with an area of about $20000 \mathrm{~km}^{2}-$ GSD $10 \mathrm{~cm}$. Including $15000 \mathrm{~km}^{2}$ under the ISOK project and $5000 \mathrm{~km}^{2}$ under the GBDOT project.

Task 4 - obtaining elements of the Topographic Object Database (BDOT) in the key scope for the implementation of flood protection tasks.

Due to the very large area of the project (92\% of Poland's area and the relatively short duration of the project, the GUGiK decided to choose INIK (Supervision and Control Inspector) an entity that will monitor the project on its behalf, organize stage acceptance, control the quality of products, and take care of for their transfer to the GUGiK (Kurczyński, Bakuła 2013). Scheme of the relations in the ISOK project are shown in the fig. 1 .

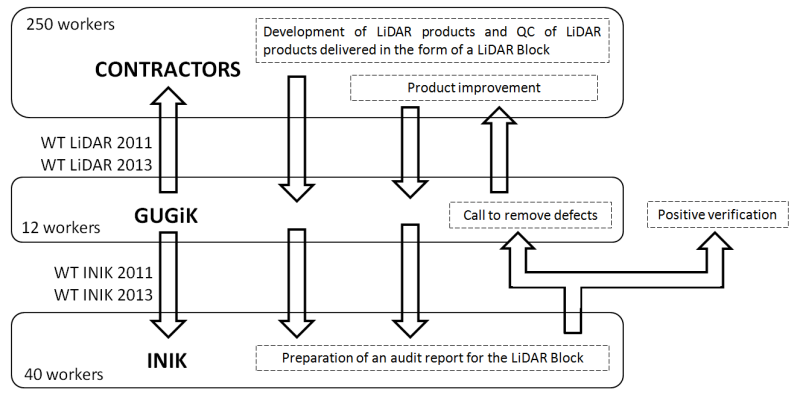

Figure 1. Scheme of the relations and information workflow in the ISOK project

The whole of INIK's activities was based on the Technical Conditions for INIK WT INiK 2011/2012, WT INiK 2013/2014 (GUGiK 2011, GUGiK 2013), in which each stage of the control was described very precisely together with the method of measuring all checked parameters.

For the needs of this project, the ALS point clouds were also acquired. In the cited documentation, the inspection stages are divided into quantitative and qualitative control. Their individual components are presented in Table 1.

Each of the points of quantitative control included in Table 1 can be also directly referred to TLS projects provided that the given features have been defined and required in the project (e.g. 1.3, 1.4 or 1.6). As for the elements of quality control, points 2.1-2.4 can be applied but also 2.5, 2.7 and 2.8 if they become due.

\begin{tabular}{|l|l|}
\hline 1 Quantitative control & 2 Quality control \\
\hline 1.1 completeness and area & 2.1 completeness of data \\
compliance of LiDAR & 2.2 data density \\
products & 2.3 uniformity of data \\
1.2 correctness of reading & 2.4 relative georeference of data \\
the saved data & 2.5 absolute georeference of data \\
1.3 correctness of file and & 2.6 block contacts \\
directory names & 2.7 data classification \\
1.4 correctness of the & 2.8 assigning RGB attributes \\
cataloging structure & 2.9 Digital Terrain Model \\
1.5 correctness of the & (DTM) \\
format and structure of the & 2.10 Digital Surface Model \\
file content & (DSM) \\
1.6 correctness of sheet cut & \\
\hline
\end{tabular}

Table 1. Stages of control in the ISOK project - ALS LiDAR data

\section{DATA QUALITY}

Due to the lack of laser scanning and BIM modeling regulations, the concept of LiDAR data evaluation has been proposed to be used in the process of developing a 3D-BIM model based on LiDAR point clouds. The standard PN-EN ISO 19157: 2014-04E "Geographical Information - Data Quality" was used for this purpose. The ISO 19157 standard provides six elements for the description of data quality. These are: completeness, logical consistency, position accuracy, thematic accuracy, time accuracy and application.

In the context of LiDAR TLS data and BIM modeling, the above elements can be adapted as follows:

1. Completeness - according to ISO standards, it is understood as the occurrence of all intended data without underflow or excess. While underflow (so-called "shadows") is a definitely negative phenomenon in the LiDAR data, it is difficult to evaluate excess unambiguously. On the one hand, scanning the same fragment several times does not increase the amount of information about the acquired object and it is economically unjustified. On the other hand, excess in a determined range, referred to as "overlaps",

is required for non-target scan techniques, such as cloud-tocloud based registration and feature based registration (Vosselman, Maas, 2010). Excess regarded as redundancy allows for calculation of fitting errors and, consequently, for accuracy control of the acquired data. Therefore, the underflow should be undoubtedly assessed as a negative phenomenon, whereas the excess treated as

a derivative of the measurement technique or redundancy will be a positive or neutral phenomenon. The most intuitive parameter will be the ratio of the area covered with LiDAR data to the whole surface expressed in $\%$.

2. Logical consistency - in (Ślusarski, 2009) we can find an explanation that logical consistency is a topological correctness of spatial data when the above concept is considered in the context of cadastral data. Due to the lack of topological dependencies in LiDAR data, one can also rely on the explanation of this concept found in (Gaździcki, 2008). The definition presented there takes into account compliance with the conceptual scheme, value domains and a format of the record as well as topological compatibility. Therefore, it is an approach similar to the scope of quantitative control (Table 1, points 1.2-1.5).

3. Position accuracy - accuracy of determining the coordinates of the point cloud. Position accuracy will consist of various 
elements depending on the assumptions of the project. It is infrequent that the contracting authority requires a point cloud and, consequently,

a model to have georeference in the state / global coordinate system. Data in the predetermined local coordinate system, on the so-called "short coordinates", are sufficient. It is also worth mentioning that despite technological advances, BIM class software often fails to deal with "long coordinates", i.e. in PL-2000 (EPSG: 2178). Therefore, if we consider the situation in the local coordinate system, the accuracy of the location of the cloud points will be influenced by the error of determining the point in

a single measurement and the error of registering all scan positions in one project. On the other hand, if georeference, i.e. "long coordinates" are required, then apart from the two previous errors one should also add the error of determining control points and the error of fitting the project into the control points. Detailed considerations in this area were presented in (Warchoł, 2018).

In the context of Table 1 point 2.4, relative georeferencing can be referred to as the accuracy of putting individual positions of the scanner into one project and it is expressed in $\mathrm{cm}$ or $\mathrm{mm}$, while the point 2.5 , that is absolute georeference also expressed in $\mathrm{cm}$ or $\mathrm{mm}$, as fitting the entire project into the determined state / global coordinate system.

4. Thematic accuracy - as we can find in (Gaździcki, 2008), it refers to the accuracy of quantitative attributes, as well as to the correctness of qualitative attributes and classification of objects and their relations. With regard to thematic accuracy, Ślusarski evokes the formulation of the correctness of determining e.g. qualitative properties. In the context of laser scanning for BIM modeling purposes, the most intuitive definition could be that thematic accuracy is a range of attributes assigned to each point of

a cloud. These can be RGB values (if during the scan images are taken and the point cloud is coloured), the intensity of the laser beam reflection, echo number, temperature values (if the scanner is equipped with a thermal camera) or classification.

5. Temporal quality - it is associated with changes of data over time and their timeliness as compared to the required frequency of updating. In case of a single data acquisition, this is the time counted from obtaining data to the present day.

6. Application - the way in which LiDAR data will be used and basically the goal of obtaining point clouds. Depending on the application, the point cloud must meet specific requirements both in terms of accuracy and density. This is especially important in determining the specification of BIM modeling based on LoD levels. In case of architecture and constructing, LiDAR data can be used to prepare models in the LoD100 - LoD300 range (fig. 2) and in case of installation in the LoD100-LoD400 range (fig. 3).

The point cloud allows for the traceability of geometry in the LoD100-LoD300 range. On the other hand, it is impossible to model fastening elements in prefabricated columns without making outcasts - LOD350 (fig. 2 c)), the same as reinforcement in LOD400 (fig. 2 d)). However, in case of installation on the LoD350 and LoD400 levels, there are additional slings and fastenings of the installation elements, i.e. objects that can be modeled from the point cloud without any problems requiring only a reasonable amount of time.

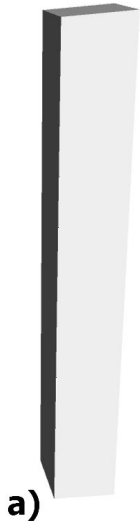

b)

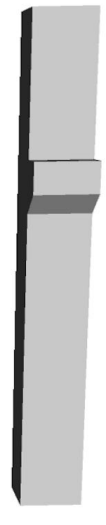

c)

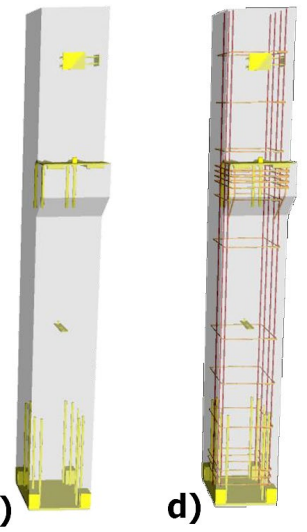

Figure 2. Example of LoD levels (Precast Structural Column Concrete): a) LoD200, b) LoD300, c) LoD350, d) LoD400

(source: BIMforum, 2019)
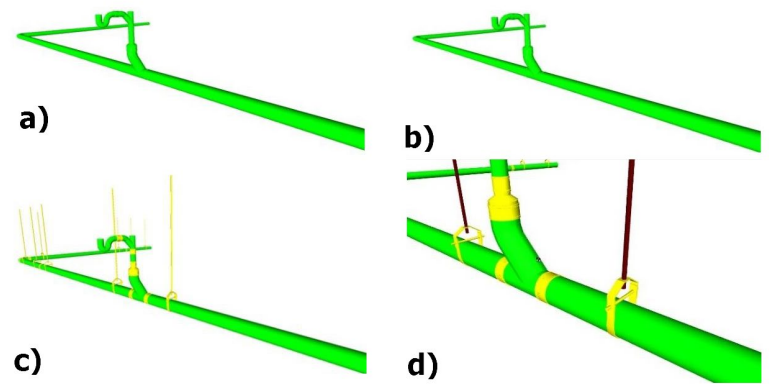

c)

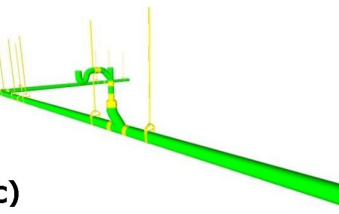

d)

Figure 3. Example of LoD levels (Sanitary Sewerage Piping): a) LoD200, b) LoD300, c) LoD350, d) LoD400 (source: BIMforum, 2019)

Another equally important aspect is the nature of the object and the scope of modeling. Different (i.e. low) cloud density is necessary to model the architecture in a newly built multifamily building (fig. 4) and completely different to develop a model of the historic facade with details and ornaments (fig. 5).

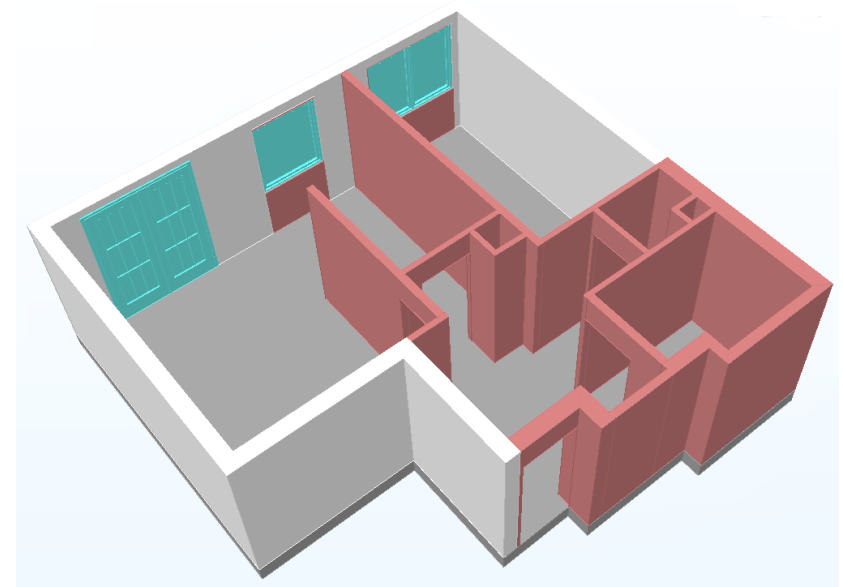

Figure. 4. Example of low detailed BIM object - newly built flat 

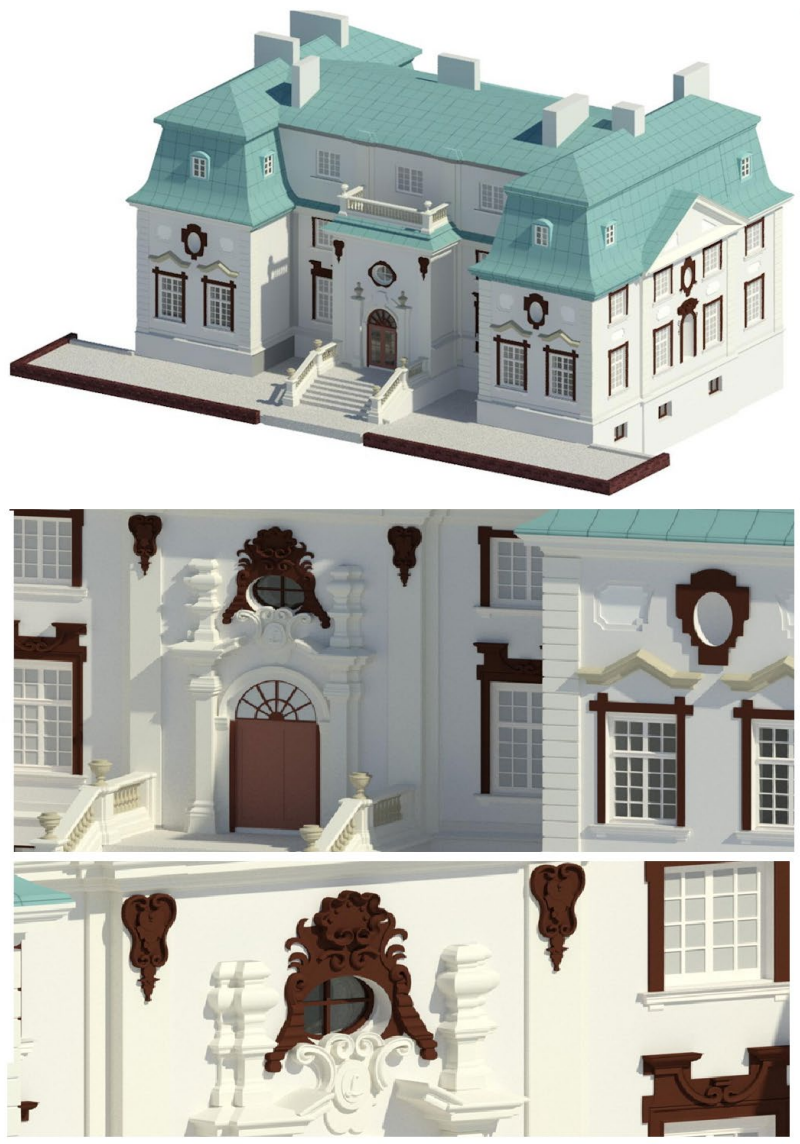

Figure 5. Example of a highly detailed BIM façade - historic building

In view of the above, it is worth adding the density of the result LiDAR data as an additional attribute characterizing the quality of the point cloud. It is important that this is the density on the object, and not the nominal one read from the manufacturer's information brochures. The issue of density was analyzed in (Warchol, 2015). The voxel approach seems to be the most complete of the approaches that are presented there. Due to the similar MLS and TLS data characteristics, it is possible to perform density measurements without filtration on the echo's number. As can be seen in table 2 in (Warchol, 2015), such approach is unauthorized with ALS data, where the density measurement described in (GUGiK, 2011 or GUGiK, 2013) should be used.

The impact of TLS data density is crucial for the recognition of individual scanned elements, and thus also for the possibility of reliable preparation on the model. On larger, uncomplicated architectural elements such as flat walls or ceilings, low density point cloud is not a problem. The large size and simple design allow reliable modeling. The situation gets more complicated when architectural details or decorations should be modeled. Examples of the influence of the resolution of the acquired point cloud on the possibilities of proper interpretation are presented below.

\section{DENSITY OF POINT CLOUDS}

In the experiment, the Faro Focus 3D S 120 scanner was used, which scanned a decorative element made of foamed polystyrene in the form of a rosette with a diameter of $0.6 \mathrm{~m}$. A photo of the test object is shown in fig. 6 .

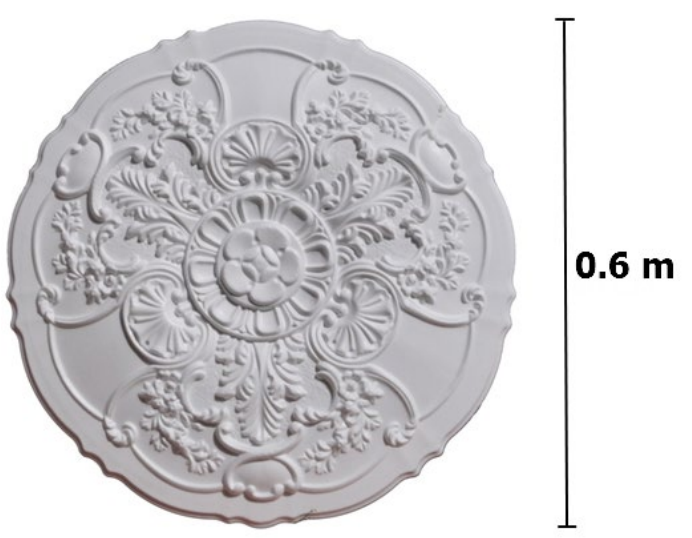

Figure 6. Test object made from styrofoam - example of a architectural detail

The model was scanned from 3 different distances: $5 \mathrm{~m}$ (fig. 7), $10 \mathrm{~m}$ (fig. 8) and $20 \mathrm{~m}$ (fig. 9). For each distance, scanning was performed at three resolution settings: $1 / 1$ (A), 1/2 (B) and 1/4 (C). Resolutions, according to the manufacturer's information, should correspond to the average distance between points: 1.534 $\mathrm{mm}(\mathrm{A}), 3.068 \mathrm{~mm}(\mathrm{~B})$ and $6.136 \mathrm{~mm}$ (B) during scanning from a distance of $10 \mathrm{~m}$. The final point clouds are shown in the figures below. Each of the point clouds is presented in a grayscale of intensity on a black background. In a situation where the cloud is more dense, the figure is "lighter" (fig. 7 A). When the data sets are more rare, the more background can be seen, making the picture darker (fig. $7 \mathrm{C}$ ). The same visualization parameters were used for all drawings.

Point clouds colored by intensity values is the best way to visualize LiDAR data.

The shape of the test object is very clearly visible in fig. $7 \mathrm{~A}$, well visible in fig. $7 \mathrm{~B}$ and fig. $8 \mathrm{~A}$ and sufficiently visible in fig. $7 \mathrm{C}$. In fig. $8 \mathrm{~B}$, most of the elements inside the model are no longer visible, while the outer contour is still clearly visible. Further increasing the distance between cloud points by increasing the scanner-object distance (fig. 9) and / or reducing the resolution causes more and more problems to identifying the object. In fig. $8 \mathrm{C}$ and fig. $9 \mathrm{~A}$ this is manifested by a barely visible edge of the detail, and in fig. $9 \mathrm{~B}$ the edge of the detail is barely visible on only $60 \%$ of the circumference. While in fig. $9 \mathrm{C}$ the test object is completely invisible.

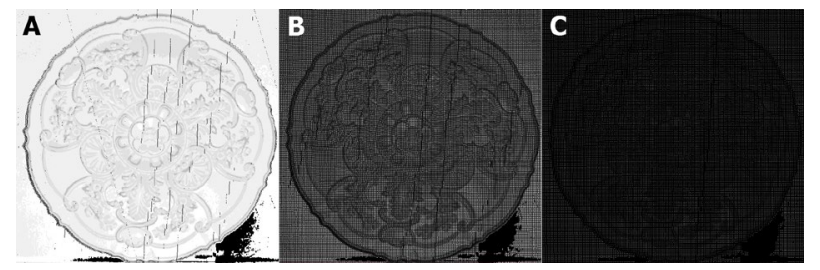

Figure 7. Point clouds of the test object, colour by intensity (greyscale) acquire from a $5 \mathrm{~m}$ distance, with resolution 1/1 (A), $1 / 2(B)$ and $1 / 4(C)$

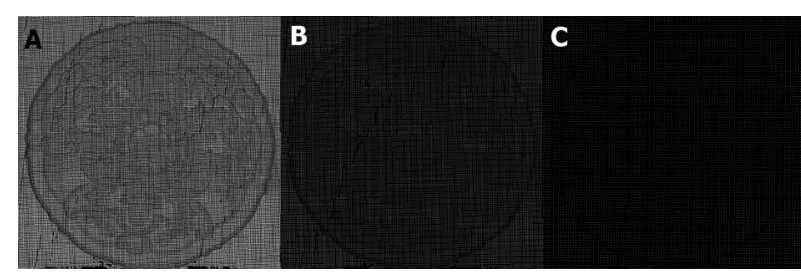

Figure 8 . Point clouds of the test object, colour by intensity (greyscale) acquire from a $10 \mathrm{~m}$ distance, with resolution $1 / 1(\mathrm{~A}), 1 / 2(\mathrm{~B})$ and $1 / 4(\mathrm{C})$ 


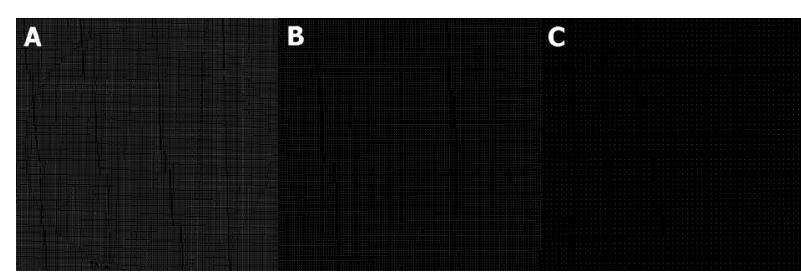

Figure 9. Point clouds of the test object, colour by intensity (greyscale) acquire from a $20 \mathrm{~m}$ distance, with resolution $1 / 1(\mathrm{~A}), 1 / 2(\mathrm{~B})$ and $1 / 4(\mathrm{C})$

To further highlight the weakness of rare point clouds, the display of the LiDAR data set has been changed from intensity to classification. The points of the cloud are more visible then, but the correct interpretation of the model shape is even more difficult. Point clouds are presented in the same scheme: $5 \mathrm{~m}$ (fig. 10), $10 \mathrm{~m}$ (fig. 11) and $20 \mathrm{~m}$ (fig. 12) and resolutions: $1 / 1(\mathrm{~A}), 1 / 2$ (B) and $1 / 4(\mathrm{C})$.

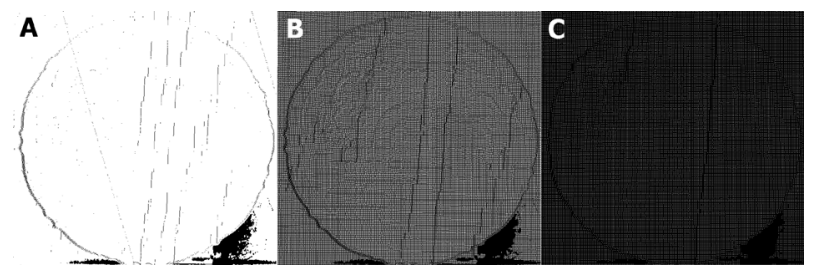

Figure 10. Point clouds of the test object, colour by classification acquire from a $5 \mathrm{~m}$ distance, with resolution $1 / 1(\mathrm{~A}), 1 / 2$ (B) and $1 / 4(\mathrm{C})$

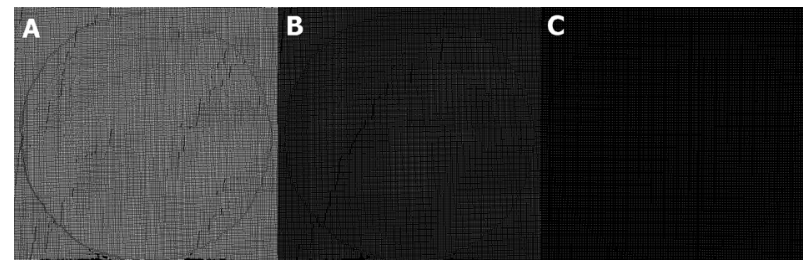

Figure 11. Point clouds of the test object, colour by classification acquire from a $10 \mathrm{~m}$ distance, with resolution $1 / 1(\mathrm{~A}), 1 / 2(\mathrm{~B})$ and $1 / 4(\mathrm{C})$

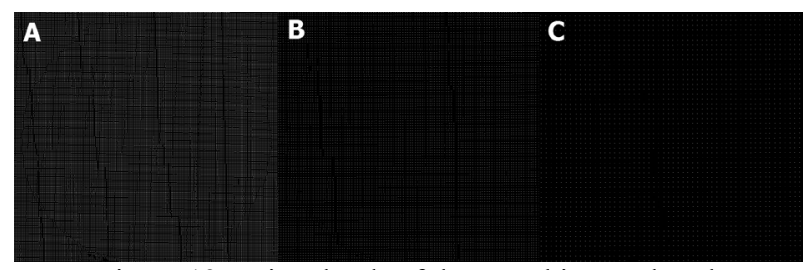

Figure 12. Point clouds of the test object, colour by classification acquire from a $20 \mathrm{~m}$ distance, with resolution $1 / 1(\mathrm{~A}), 1 / 2(\mathrm{~B})$ and $1 / 4(\mathrm{C})$

As can be seen in the figures above, displaying only point cloud geometry makes it difficult to correctly interpret the modelled object.

\section{CONCLUSIONS}

Data information, i.e. metadata, is an extremely important part of any data set. Especially for such large sets, which are point clouds, whose volumes usually start from a few GB and end on a few TB.

In order to facilitate the assessment of the suitability of a particular data set for various applications, it is worth having a metric for each LiDAR data sets. Based on the ISO 19157 standard and own experience, a list of attributes was proposed which should be included in the LiDAR-TLS metric. Not all attributes are equally needed, therefore the proposed list has priorities: 1 - highest priority (must be) to 5 - lowest priority (not necessary).

\begin{tabular}{|c|c|}
\hline Attribute & $\begin{array}{c}\text { Priority } \\
(\mathbf{1} \text { - the highest } \\
\text { 5 - the lowest })\end{array}$ \\
\hline Completeness & 2 \\
\hline Logical consistency & 3 \\
\hline Position accuracy & 1 \\
\hline Thematic accuracy & 3 \\
\hline Temporal quality & 4 \\
\hline Application & 5 \\
\hline Density & 1 \\
\hline
\end{tabular}

Table 2. Proposed priority list of attributes of the point clouds in the BIM modeling context

\section{ACKNOWLEDGEMENTS}

Thank you M.Sc. Joanna Kołodziej for help in the area of acquiring point clouds for the experiment.

\section{REFERENCES}

BIMforum, 2019. Level of Development (LOD) Specification. ver. April 2019. https://bimforum.org/lod/ (31 July 2019)

Gaździcki, J., 2008. Geomatic lexicon. www.ptip.org.pl. (31 July 2019)

GUGiK, 2011. Technical conditions for the implementation of the service consisting in the performance of support tasks and control tasks related to the procurement carried out by ISOK Contractors under the order "Service of data verification and coordination of activities under the ISOK project". Annex No. 1 to the Terms of Reference - WT INiK.

GUGiK, 2013. Technical conditions for the implementation of the data verification service as part of the ISOK - INiK3 project. Annex No. 1 to the Terms of Reference - Detailed Description of the Object of the Contract - WT INiK3.

Kurczyński Z., Bakuła K., 2013. Generation of countrywide reference Digital Terrain Model from Airborne Laser Scannig in ISOK project. Archives Of Photogrammetry, Cartography And Remote Sensing special issue: "Measurement Technologies in Surveying" pp.59-68 ISBN 978-83-61576-26-7

PN-EN ISO 19157:2014-04 - Geographic Information - Data Quality

Ślusarski, M., 2009. Methodology to describe the quality of spatial data in the aspect of ISO standards. ISTCGCAP. Vol. 71, Number 71: pp. 136-139. http://science.lpnu.ua/istcgcap/allvolumes-and-issues/volume-71-2009/metodyka-opisu-jakoscidanych-przestrzennych-w (31 July 2019)

Warchoł A., 2015. Density of point clouds in Mobile Laser Scanning. Archives Of Photogrammetry, Cartography And Remote Sensing. vol. 27, s. 149-161. DOI: 10.14681/afkit.2015.011

Warchoł, A., 2018. Selected aspects of modeling objects based on LIDAR data in the aspect of BIM. Presentation on XXVII SCIENTIFIC AND TECHNICAL CONFERENCE "Geodesy, 
The International Archives of the Photogrammetry, Remote Sensing and Spatial Information Sciences, Volume XLII-1/W2, 2019

Evaluation and Benchmarking Sensors, Systems and Geospatial Data in Photogrammetry and Remote Sensing, 16-17 Sept. 2019, Warsaw, Poland

construction, environment - scientific, legal, practical and didactic aspects - 8-9.05.2018 Rzeszow

Vosselman, G., Maas, H-G., 2010. Airborne and Terrestrial Laser Scanning. CRC Press 